\title{
The association of levels of physical activity with metabolic syndrome in rural Australian adults
}

\author{
Clare Vaughan ${ }^{* 11}$, Adrian Schoo ${ }^{\dagger 1}$, Edward D Janus ${ }^{\dagger 2}$, Benjamin Philpot $^{\dagger 1}$, \\ Nathalie Davis-Lameloise ${ }^{\dagger 1}$, Sing Kai Lo ${ }^{\dagger 3}$, Tiina Laatikainen ${ }^{\dagger 4}$, \\ Erkki Vartiainen ${ }^{\dagger 4}$ and James A Dunbar ${ }^{\dagger 1}$
}

\author{
Address: ${ }^{1}$ Greater Green Triangle University Department of Rural Health, A partnership between Flinders and Deakin Universities, PO Box 423 , \\ Warrnambool, 3280, Victoria, Australia, ${ }^{2}$ Department of Medicine, University of Melbourne, Western Hospital, Footscray, 3011, Victoria, \\ Australia, ${ }^{3}$ Faculty of Health, Medicine, Nursing and Behavioural Science, Deakin University, Burwood, 3125, Victoria, Australia and ${ }^{4}$ Division of \\ Welfare and Health Promotion, National Institute for Health and Welfare (THL), Mannerheimintie 166, 00300 Helsinki, Finland \\ Email: Clare Vaughan* - clare.vaughan@greaterhealth.org; Adrian Schoo - adrian.schoo@greaterhealth.org; \\ Edward D Janus - edwarddj@unimelb.edu.au; Benjamin Philpot - ben.philpot@greaterhealth.org; Nathalie Davis- \\ Lameloise - nathalie.davis@greaterhealth.org; Sing Kai Lo - singkai.lo@deakin.edu.au; Tiina Laatikainen - tiina.laatikainen@thl.fi; \\ Erkki Vartiainen - erkki.vartiainen@thl.fi; James A Dunbar - director@greaterhealth.org \\ * Corresponding author †Equal contributors
}

Published: 31 July 2009

BMC Public Health 2009, 9:273 doi:10.1 186/147/-2458-9-273

This article is available from: http://www.biomedcentral.com/147/-2458/9/273

(c) 2009 Vaughan et al; licensee BioMed Central Ltd.

This is an Open Access article distributed under the terms of the Creative Commons Attribution License (http://creativecommons.org/licenses/by/2.0), which permits unrestricted use, distribution, and reproduction in any medium, provided the original work is properly cited.
Received: 17 December 2008

Accepted: 31 July 2009

\begin{abstract}
Background: Physical activity (PA) reduces risk factors related to metabolic syndrome. Rurality influences the way people incorporate physical activity into daily life. The aim of this study is to determine the association of PA level with metabolic syndrome in a rural Australian population. The influence of adiposity on these associations is also investigated.

Methods: Three cross-sectional population health surveys were conducted in south-east Australia during 2004-2006 using a random population sample ( $n=1563$, participation rate 49\%) aged 2574 years. PA was assessed via a self-administered questionnaire, and components of the metabolic syndrome via anthropometric measurements taken by specially trained nurses and laboratory tests.

Results: Approximately one-fifth of participants were inactive in leisure-time and over one-third had metabolic syndrome (men $39 \%$, women $33 \% ; p=0.022$ ). There was an inverse association between level of PA and metabolic syndrome $(P<0.001)$. Men who were inactive in leisure-time were more than twice as likely and women more than three times as likely to have metabolic syndrome compared with those having high PA. Body mass index (BMI) is a mediating factor in the association between level of PA and metabolic syndrome.
\end{abstract}

Conclusion: Some PA is better than none if adults, particularly women, are to reduce their risk of metabolic syndrome and associated vascular diseases. Specialised interventions that take rurality into consideration are recommended for adults who are inactive. 


\section{Background}

Regular physical activity (PA) has a mild or moderate effect on numerous metabolic and cardiovascular risk factors that constitute or are related to the metabolic syndrome [1]. Metabolic syndrome includes a clustering of abnormal components: abdominal obesity, fasting hyperglycemia, hypertension and dyslipidaemia (high triglycerides, low high-density lipoprotein (HDL) cholesterol). By identifying people with metabolic syndrome, irrespective of the definition used [2,3], individuals who are at increased long-term risk of type 2 diabetes mellitus (T2DM) and cardiovascular disease (CVD) can be identified, and this provides opportunity for prevention through lifestyle intervention $[4,5]$.

Lifestyle intervention to reduce risk of vascular diseases is mainly through diet and PA modification. A contemporary position on optimal levels of PA for health benefits promotes incidental activity in conjunction with 30 minutes of moderate-intensity activity on most days of the week which includes muscle strengthening activities [6]. The guidelines do not caution against sedentariness however the case is building for this [7]. When measuring PA levels, some studies measure total PA while others measure the domains of leisure-time, occupational or commuting PA separately. A previous investigation of the current study sample reported that few of the rural Australian adults met the level of PA needed for health benefits; most were moderately active in leisure-time; the majority of men engaged in high-level occupational PA (mainly employed in agriculture, forestry and fishing) with women reporting mostly low-level PA occupations (such as administration, management, education and other services); and approximately one-third of participants actively commuted to work [8]. It was hypothesised that with mechanisation of rural occupations, men perceive they are more active than in reality. It was also suggested that few people actively commute to work because the distances regularly travelled are beyond the 5-10 kilometres suited to active commuting [9] and infrastructure to safely separate active commuters from motorised vehicles is limited. Leisure-time physical activity (LTPA) is defined in this study as voluntary and purposeful activity, carried out to improve one or more components of physical fitness. LTPA is the preferred measure for this study because the categories include a comprehensive list of examples from sport and recreation, home duties and gardening as well as walking and cycling.

Strong evidence exists to show that obesity is a major risk factor for metabolic syndrome [10] and low levels of PA contribute to overweight and obesity [11]. There is increasing evidence from population studies describing an inverse association between level of PA and the prevalence of metabolic syndrome [12-21], however, to the best of our knowledge none of these studies are rural nor do they make clear the influence of overweight and obesity on the association. The aim of this study is to investigate the association of level of LTPA with metabolic syndrome in a random sample of adults from a rural Australian population. The influence of adiposity on the association is also investigated.

\section{Methods \\ Participants}

Three cross-sectional population health surveys were carried out in the Greater Green Triangle (GGT) region of south-east Australia between October 2004 and October 2006. The region is predominantly agricultural with multiple small towns of less than 1000 people and the two largest towns have 23,000 and 13,000 residents. Independent age and sex stratified random samples of people aged 25 to 74 years were drawn from the electoral roll. The total sample was 1563 participants with a $49 \%$ response rate.

\section{Data collection and measurements}

The survey methodology closely followed the WHO MONICA protocol [22] and recommendations from the European Health Risk Monitoring Project [23]. The surveys were comprised of a self-administered questionnaire which included questions related to level of PA, plus a series of anthropometric measurements and laboratory tests to quantify cardiovascular and metabolic risk factors. The questionnaire, together with an invitation to attend a health check, was sent by mail to all selected participants and nurses specially trained in the survey procedures conducted the measurements; an explanation of these processes has been described elsewhere [24]. The protocols and procedures for measuring height, weight, waist circumference and blood pressure, for computing body mass index (BMI), and for venous blood sampling to determine fasting blood glucose and lipid profile are also described in detail elsewhere [24].

LTPA was categorised as 1) high: participation in activities to maintain fitness (running, jogging, gymnastics, swimming, ball-games or heavy gardening) or regular training for competitions several days a week; 2) low to moderate: walking, cycling or participating in another form of light exercise (fishing, home duties, light gardening) for at least four hours per week; and 3) inactive: reading, watching TV or other passive pursuits.

Metabolic syndrome was defined in this study according to the International Diabetes Federation (IDF) definition, which is suitable for all ethnic groups [2]. In addition, the definition of the Third Report of the National Cholesterol Education Program Expert Panel on Detection, Evaluation, and Treatment of High Blood Cholesterol in Adults, 
Adult Treatment Panel III (NCEP ATP III) was used for the analysis [3]. The definitions are:

a) IDF criteria specify central obesity with waist circumference $\geq 94 \mathrm{~cm}$ for men and $\geq 80 \mathrm{~cm}$ for women of Europid origin, plus two or more of the following: systolic blood pressure $\geq 130 \mathrm{mmHg}$ or diastolic blood pressure $\geq 85$ mmHg or antihypertensive medication; fasting plasma glucose $\geq 5.6 \mathrm{mmol} / \mathrm{L}$ or previously diagnosed type 2 diabetes; plasma HDL-cholesterol $<1.03 \mathrm{mmol} / \mathrm{L}$ for men and $<1.29 \mathrm{mmol} / \mathrm{L}$ for women; plasma triglycerides $\geq 1.7$ $\mathrm{mmol} / \mathrm{L}$.

b) NCEP ATP III criteria includes three or more of the following: waist circumference $\geq 102 \mathrm{~cm}$ for males and $\geq 88$ $\mathrm{cm}$ for females; systolic blood pressure $\geq 130 \mathrm{mmHg}$ or diastolic blood pressure $\geq 85 \mathrm{mmHg}$ or antihypertensive medication; fasting plasma glucose $\geq 5.6 \mathrm{mmol} / \mathrm{L}$ or on medication for high blood glucose; HDL cholesterol $<1.03 \mathrm{mmol} / \mathrm{L}$ for males and $<1.30 \mathrm{mmol} / \mathrm{L}$ for females; triglycerides $\geq 1.7 \mathrm{mmol} / \mathrm{L}$.

Ethics approval was received from Flinders University Clinical Research Ethics Committee. Informed consent was received from participants when they attended the clinical testing component of the survey.

\section{Statistical analyses}

Statistical analyses were undertaken using Stata version 10.1. Two-sided independent t-tests and Pearson chisquare tests were used to investigate gender differences in study population characteristics (Table 1 ), and the association between LTPA and metabolic syndrome (Table 2). For further analyses, data were weighted by the age and sex distribution of the survey regions according to the electoral roll.

Linear regression was used to compare the means of BMI, waist circumference, systolic blood pressure (SBP), diastolic blood pressure (DBP), fasting plasma glucose, HDLcholesterol, and triglycerides among participants in the three LTPA levels (Additional file 1). The mean differences between LTPA levels and 95\% confidence intervals are presented for males and females separately.

Three models were constructed. Model one included the covariates: study area, age, education, smoking status and alcohol consumption. The second model included BMI in addition to the above covariates whereas the third model included waist circumference instead of BMI in addition to the above covariates listed.

For the logistic regression analyses, dichotomous variables were created using the IDF criteria of metabolic syndrome and its components, in addition to BMI (cut-off 30 $\mathrm{kg} / \mathrm{m}^{2}$ ) and the NCEP ATP III criteria of metabolic syndrome (Additional file 2). Odds ratios and 95\% confidence intervals are presented for the 3 models as described above.

Table I: Characteristics of study population $[n, \%]$

\begin{tabular}{|c|c|c|c|c|c|}
\hline \multirow[b]{2}{*}{$\mathrm{n}$} & \multicolumn{2}{|l|}{ Male } & \multicolumn{2}{|c|}{ Female } & \multirow[t]{2}{*}{ P-value } \\
\hline & 721 & 46.8 & 818 & 53.2 & \\
\hline Age (mean, SD) & 55.8 & 12.1 & 55.0 & 11.7 & 0.182 \\
\hline Education years (mean, SD) & 11.2 & 2.9 & 11.4 & 2.7 & 0.169 \\
\hline Leisure-time PA level & & & & & $<0.001$ \\
\hline Inactive & 143 & 19.8 & 123 & 15.0 & \\
\hline Low/moderate & 451 & 62.6 & 602 & 73.6 & \\
\hline High & 127 & 17.6 & 93 & 11.4 & \\
\hline Cardiovascular Disease & 87 & 12.1 & 52 & 6.4 & $<0.001$ \\
\hline \multicolumn{6}{|l|}{ Vascular risk factors } \\
\hline Standard alcohol drinks per week (mean, SD) & 10.8 & 14.2 & 3.8 & 5.8 & $<0.001$ \\
\hline Current smoker & 126 & 17.5 & 108 & 13.4 & \\
\hline Ex-smoker & 274 & 38.1 & 215 & 26.7 & \\
\hline Non-smoker & 319 & 44.4 & 483 & 59.9 & \\
\hline BMI $\left(\geq 30 \mathrm{~kg} / \mathrm{m}^{2}\right)$ & 202 & 30.3 & 255 & 35.0 & 0.062 \\
\hline LDL-cholesterol (>2.5 mmol/L or on lipid lowering medication) & 592 & 89.8 & 618 & 84.8 & 0.005 \\
\hline Metabolic Syndrome (IDF) & 250 & 39.0 & 232 & 33.0 & 0.022 \\
\hline Waist circumference ( $\leq 102 \mathrm{~cm}$ for males and $\leq 88 \mathrm{~cm}$ for females) & 453 & 68.3 & 529 & 72.6 & 0.083 \\
\hline Hypertension ( $\geq 130 / 85$ or on antihypertensive medication) & 439 & 64.4 & 434 & 57.1 & 0.005 \\
\hline Fasting plasma glucose $(\geq 5.6 \mathrm{mmol} / \mathrm{L}$ or on an antidiabetic medication) & 215 & 33.3 & 144 & 20.4 & $<0.001$ \\
\hline HDL-cholesterol (males < $1.03 \mathrm{mmol} / \mathrm{L}$; females < $1.30 \mathrm{mmol} / \mathrm{L}$ ) & 154 & 23.6 & 126 & 17.9 & 0.009 \\
\hline Triglycerides ( $\geq 1.7 \mathrm{mmol} / \mathrm{L})$ & 224 & 35.5 & 200 & 29.0 & 0.012 \\
\hline
\end{tabular}

Cardiovascular disease includes stroke, myocardial infarction, coronary bypass surgery or coronary angioplasty 
Table 2: Prevalence of metabolic syndrome (IDF, NCEP ATP III) and leisure-time physical activity

\begin{tabular}{lllll}
\hline & & IDF & \multicolumn{3}{c}{ NCEP ATP III \# } \\
\hline LTPA level & $\mathrm{n}$ & $\%$ & $\mathrm{n}$ & $\%$ \\
Inactive & $\mathrm{II}$ & 48.9 & 100 & 43.7 \\
Low/moderate & 327 & 35.7 & 280 & 30.7 \\
High & 43 & 21.5 & 36 & 18.1 \\
Overall & 482 & 35.9 & 862 & 64.1
\end{tabular}

\# Level of leisure-time PA and metabolic syndrome $(p<0.00$ I)

\section{Result}

This study included 1539 rural adults (men 47\%, women $53 \%$ ) aged 25-74 years with data available for LTPA (Table 1 ). The majority of participants reported a low to moderate level of LTPA (68\%) and nearly one-fifth were inactive in leisure-time. Over one-third of participants had metabolic syndrome (men 39\%, women 33\%; p = 0.022 ; Table 1). The prevalence of hypertension and the concentrations of fasting plasma glucose, HDL-cholesterol and triglycerides based on the IDF cut-off points were worse in men than in women (Table 1). The prevalence of BMI and waist circumference above the cut-off points were not significantly different between men and women. There was an inverse association between level of LTPA and metabolic syndrome based on IDF and NCEP ATP III criteria ( $<<0.001)$ (Table 2).

Among men and women, there was a significant association between the three levels of LTPA and waist circumference the inactive group had the highest waist circumference and the high LTPA group the lowest (Additional file 1, model 1). Similar associations were generally found for LTPA and BMI. After BMI adjustment (Additional file 1, model 2), the association remained for waist circumference in men and not women. After adjustment for waist circumference (Additional file 1, model 3), the association with BMI was no longer significant for any group.

In women, the other components of metabolic syndrome significantly associated with level of LTPA were fasting plasma glucose, HDL cholesterol and triglycerides (Additional file 1, model 1). After adjustment for the effect of BMI (Additional file 1, model 2), and waist circumference (Additional file 1, model 3), significant associations remained for fasting plasma glucose.

Men who were inactive in leisure-time were more than twice as likely and women nearly four times more likely to have metabolic syndrome based on IDF inclusion criteria, compared with those having high LTPA (Additional file 2, model 1). Similar four-fold increases in probability of having metabolic syndrome were found for women, when data were analysed using the NCEP ATP III definition (Additional file 2, model 1).

Men and women who were inactive in leisure-time were approximately four times more likely to be obese (BMI $\geq$ $30 \mathrm{~kg} / \mathrm{m}^{2}$ ) compared with those having high LTPA (Additional file 2, model 1); similar results were found for central obesity (waist circumference based on IDF cut-offs). Men with low to moderate LTPA were more likely than inactive men to have the fasting plasma glucose and triglyceride components of metabolic syndrome above cutoff points (Additional file 2, models 2 and 3). In contrast, women who were inactive in leisure-time were over three times more likely to have fasting plasma glucose or triglycerides components of metabolic syndrome above the IDF cut-offs compared with those having high LTPA.

\section{Discussion}

To our knowledge, this study is the first to describe the inverse association between LTPA and metabolic syndrome in a randomly selected rural adult population. Both men and women who were inactive in leisure-time were more likely to have metabolic syndrome compared with those who had high LTPA. This was a robust finding based on the IDF definition of metabolic syndrome. Using the NCEP ATP III definition, the significant association was found for women only. We consider the IDF definition of metabolic syndrome more appropriate since central obesity is a defined component and there are ethnicity specific values. By contrast, the NCEP ATP III definition, for example, could include thin Asian people with hypertension and dyslipidaemia along with obese individuals [25].

The inverse association between LTPA and metabolic syndrome has previously been reported [12-21], however those studies either used the NCEP ATP III definition of metabolic syndrome, or were conducted in selected populations. Even though a range of instruments are used to measure PA, the relationships with metabolic syndrome have nevertheless been consistent. Currently, there is no gold standard for self-reported physical activity measurement [26]. The tool used in this study is a simple selfreported measure suited to the self-administered questionnaire format, and it has been used and reported elsewhere [27].

The reported findings on the influence of rurality on metabolic syndrome and its key component obesity are inconsistent [28-31]. As part of the phenomenon of epidemiological transition, developing countries tend to show urban obesity, and developed countries tend to show rural obesity. In Australia, there is some suggestion that rural and urban areas have a similar prevalence of obesity [24]. 
Several studies, one of which was conducted in a 'realworld' setting in rural Australia, have highlighted the importance of weight loss, particularly through reduced waist circumference as a primary target for prevention of T2DM [32-34]. It is of concern that one-fifth of participants in this study were inactive in leisure-time and that this group was most likely to be obese and have waist circumferences exceeding the IDF cut-off points. Our results show an association between obesity (BMI and waist circumference) and LTPA which is consistent with another Finnish population study that used the same tool as our study to measure LTPA [27]. To determine whether BMI and waist circumference were confounding factors in the relationship between LTPA and metabolic syndrome, we adjusted for the effect of these variables (Additional file 2 ). Our results suggest that BMI and waist circumference are mediating factors in the relationship, particularly for women and weight loss is likely to reduce risk of metabolic syndrome and therefore T2DM and CVD.

One of the strengths of the study is the random sample drawn from a rural adult population. Although the participation rate was modest (49\%), a comparison of socioeconomic characteristics of survey participants with population statistics available indicated that participants closely represented the true populations of the areas [35]. Another strength is that a close comparison could be made with the study by Barengo et al. [27] since it used the same LTPA measure and similar methods of analysis. The present study was enhanced by the investigation of the relationships between LTPA and metabolic syndrome and its components.

A limitation of this study is that it relies on self-reported LTPA. Self-reported physical activity measures are associated with over-reporting [26] however such measures are widely used as indicators of activity, and the costs of more objective physical measures were beyond the scope of this study. Dietary habits were also self-reported in the survey; however there was no overall measure to include as a covariate in the analyses. Another limitation is the disparities found in men for fasting plasma glucose and triglycerides which remain unexplained. The result of no effect was also found in another Australian study, where the positive association between sedentary behaviour and fasting plasma glucose or triglyceride was found for women and not men [36].

The greatest public health gains from physical activity promotion come from increasing the physical activity level of people who are sedentary [11]. It has been suggested that in rural Australia, facilities and opportunities for LTPA and active commuting tend to be limited compared with urban areas as a result of smaller and more dispersed populations. Unstructured or low-intensity activity such as walking has been shown to reduce the risk of developing metabolic syndrome [37], and tai chi has shown promise [38]. These activities do not require specialist facilities and are suited to a low intensity start-point with gradual progressions making them appropriate for inactive adults living in rural areas. Active obese individuals have lower cardiovascular disease risk than those who are sedentary and obese [39], and the types of activities suggested above are also appropriate for rural obese people. The challenge for health professionals is how to best intervene with this difficult to reach group.

Health professionals working in rural areas require specialised skills to support people who may not perceive themselves as inactive, to plan for, and engage in LTPA. Rural health professionals require an understanding of occupational physical activity in the rural context as well as the challenges of active commuting in these regions and the limited opportunities and facilities for LTPA. In 2006, a project conducted in the same region of rural Australia as the current study, reported on an effective group lifestyle modification program designed for people at high risk of developing T2DM [33]. More women than men chose to participate in the program and the results of this study suggest that women are particularly vulnerable to cardiovascular disease if they are inactive. Program success was attributed to factors that include the theory based health behaviour principles that underpinned the program and the fact that local health professionals who delivered the program were trained in non-directive counselling techniques and group facilitation. The group program mentioned above supports these processes and the group helped to generate possible solutions that individuals could adopt for themselves. The program is particularly suited to increase LTPA in inactive adults with metabolic syndrome who live in rural areas.

\section{Conclusion}

At least 20 percent of rural Australian adults in this study were physically inactive in their leisure-time and this inactivity was associated with an increased probability of having metabolic syndrome, particularly in women. Some physical activity is better than none if adults are to reduce their risk of metabolic syndrome and other vascular diseases.

\section{Competing interests}

The authors declare that they have no competing interests.

\section{Authors' contributions}

$\mathrm{CV}$ coordinated writing the manuscript. AS initiated the article and contributed to writing the manuscript. EDJ initiated, organised and supervised the surveys, contributed to data interpretation and writing the manuscript. BP conducted the statistical analysis and contributed to writing 
the manuscript. NDL contributed to writing the manuscript. SKL provided expert guidance in manuscript planning and statistical analysis. TL initiated, organised and supervised the surveys, contributed to data interpretation and writing the manuscript. EV was involved in the survey design and contributed to data analysis. JAD was the lead investigator in the three surveys, is the guarantor and contributed to writing the manuscript. All authors read and approved the final version of the manuscript.

\section{Additional material}

\section{Additional file 1}

Supplemental Table. Mean differences in cardiovascular and metabolic risk factors according to level of leisure-time PA among rural Australian adult

Click here for file

[http://www.biomedcentral.com/content/supplementary/14712458-9-273-S1.doc]

\section{Additional file 2}

Supplemental table. Indicators of elevated cardiovascular and metabolic risk factors according to leisure-time PA level among adults in a rural Australian population

Click here for file

[http://www.biomedcentral.com/content/supplementary/14712458-9-273-S2.doc]

\section{Acknowledgements}

Stephen Bunker, Andrew Baird, Anna Chapman, Anna Kao-Philpot, and the nurses carrying out the survey and regional hospitals providing facilities for the study.

This study was supported by the Australian Government Department of Health and Ageing, Royal Australian College of General Practitioners, Sanofi-Aventis PL, Pfizer Inc, Roche Diagnostics and Servier Laboratories Australia PL.References

\section{References}

I. Lakka TA, Laaksonen DE: Physical activity in prevention and treatment of the metabolic syndrome. Appl Physiol Nutr Me 2007, 32(I):76-88.

2. Alberti KG, Zimmet P, Shaw J: Metabolic syndrome-a new worldwide definition. A Consensus Statement from the International Diabetes Federation. Diabet Med 2006, 23(5):469-480.

3. National Cholesterol Education Program (NCEP) Expert Panel: Third Report on the Detection, Evaluation, and Treatment of High Blood Cholesterol in Adults. Circulation 2002, I 06(25):3|43-342|

4. Ilanne-Parikka P, Eriksson JG, Lindstrom J, Peltonen M, Aunola S, Hamakainen $\mathrm{H}$, Keinanen-Kiukaanniemi S, Laakso M, Valle TT, Lahtela $\mathrm{J}$, et al:: Effect of lifestyle intervention on the occurrence of metabolic syndrome and its components in the Finnish Diabetes Prevention Study. Diabetes Care 2008, 3 I (4):805-807.

5. Orchard TJ, Temprosa M, Goldberg R, Haffner S, Ratner R, Marcovina $S$, Fowler S: The effect of metformin and intensive lifestyle intervention on the metabolic syndrome: the Diabetes Prevention Program Randomized Trial. Ann Intern Med 2005, |42(8):6 I I.

6. Department of Human Services and Health: Physical Activity Guidelines for Americans 2008. [http://www.health.gov/ PAGuidelines/pdf/paguide.pdf]. Accessed May 2009
7. Brown W, Bauman AE, Owen N: Stand up, sit down, keep moving: turning circles in physical activity research? $\mathrm{Br} J$ Sports Med 2008, 43:86-88.

8. Vaughan C, Kilkkinen A, Philpot B, Brooks J, Schoo A, Laatikainen T, Chapman A, Janus ED, Dunbar JA: Physical activity behaviours of adults in the Greater Green Triangle region of rural Australia. AJRH 2008, I 6(2):92-99.

9. Rissel C, Garrard J: Cycling for active transport and recreation in Australia: status review and future directions. World Transport Policy and Practice 2006, I 3(I):49-63.

10. Nguyen NT, Magno CP, Lane KT, Hinojosa MW, Lane JS: Association of Hypertension, Diabetes, Dyslipidemia, and Metabolic Syndrome with Obesity: Findings from the National Health and Nutrition Examination Survey, 1999 to 2004. J Am Coll Surg 2008, 207(6):928-934.

II. Haskell WL, Lee IM, Pate RR, Powell KE, Blair SN, Franklin BA, Macera CA, Heath GW, Thompson PD, Bauman A: Physical Activity and Public Health: Updated Recommendation for Adults from the American College of Sports Medicine and the American Heart Association. Med Sci Sport Exe 2007, 39(8): | 423-|434.

12. Franks PW, Ekelund U, Brage Sr, Wareham NJ, Wong M-Y: Does the Association of Habitual Physical Activity With the Metabolic Syndrome Differ by Level of Cardiorespiratory Fitness? Diabetes Care 2004, 27(5): I I87-I I 94.

13. Halldin M, Rosell M, de Faire U, Hellénius ML: The metabolic syndrome: Prevalence and association to leisure-time and workrelated physical activity in 60-year-old men and women. Nutr Metab Cardiovas 2007, I 7(5):349-357.

14. Irwin ML, Ainsworth BE, Mayer-Davis EJ, Addy CL, Pate RR, Durstine $\mathrm{JL}$ : Physical activity and the metabolic syndrome in a tri-ethnic sample of women. Obes Res 2002, I 0(I 0): 1030.

I5. Kullo IJ, Hensrud DD, Allison TG: Relation of low cardiorespiratory fitness to the metabolic syndrome in middle-aged men. Am J Cardiol 2002, 90(7):795-797.

16. Lakka TA, Laaksonen DE, Lakka HM, Mannikko N, Niskanenl LK, Rauramaa R, Salonen JT: Sedentary Lifestyle, Poor Cardiorespiratory Fitness, and the Metabolic Syndrome. Med Sci Sports Exerc 2003, 35(8): I 279-1286.

17. Mohan V, Gokulakrishnan K, Deepa R, Shanthirani CS, Datta M: Association of physical inactivity with components of metabolic syndrome and coronary artery disease; the Chennai Urban Population Study (CUPS no. I5). Diabetic Med 2005, 22(9): |206-|2||.

18. Rennie KL, McCarthy N, Yazdgerdi S, Marmot M, Brunner E: Association of the metabolic syndrome with both vigorous and moderate physical activity. Int J Epidemiol 2003, 32(4):600-606.

19. Wilsgaard T, Jacobsen BK: Lifestyle factors and incident metabolic syndrome: The Tromsø Study 1979-200 I. Diabetes Res Clin Pr 2007, 78(2):217-224.

20. Yang X, Telama R, Hirvensalo M, Mattsson N, Viikari JS, Raitakari OT: The longitudinal effects of physical activity history on metabolic syndrome. Med Sci Sports Exerc 2008, 40(8):|424-|43|.

21. Panagiotakos DB, Pitsavos C, Chrysohoou C, Skoumas J, Tousoulis D, Toutouza M, Toutouzas P, Stefanadis C: Impact of lifestyle habits on the prevalence of the metabolic syndrome among Greek adults from the ATTICA study. Am Heart J 2004, I47(I): $106-112$.

22. WHO MONICA Project Principal Investigators: The World Health Organization MONICA Project (monitoring trends and determinants in cardiovascular disease): a major international collaboration. J Clin Epidemiol 1988, 41: I05-I |4.

23. Tolonen H, Kuulasmaa K, Laatikainen T, Wolf H: European Health Risk Monitoring Project. Recommendation for indicators, international collaboration, protocol and manual of operations for chronic disease risk factor surveys. EHRM Project 2002 [http://www.ktl.fi/publications/ehrm/product2/title.htm]. Accessed May 2009

24. Janus ED, Laatikainen T, Dunbar JA, Kilkkinen A, Bunker SJ, Philpot B, Tideman PA, Tirimacco R, Heistaro S: Overweight, obesity and metabolic syndrome in rural southeastern Australia. MJA 2007, I 87(3): | 47.

25. Patel A, Huang KC, Janus ED, Gill T, Neal B, Suriyawongpaisal P, Wong $E$, Woodward $M$, Stolk RP: Is a single definition of the metabolic syndrome appropriate? A comparative study of the USA and Asia. Atherosclerosis (002/9/50) 2006, I 84(I):225-232. 
26. Bauman AE: Updating the evidence that physical activity is good for health: an epidemiological review 2000-2003. J Sci Med Sport 2004, 7(I Suppl):6-19.

27. Barengo N, Kastarinen M, Lakka T, Nissinen A, Tuomilehto J: Different forms of physical activity and cardiovascular risk factors among 24-64-year-old men and women in Finland. Eur J Cardiov Prev R 2006, I 3(I):5I-59.

28. Bouguerra R, Ben Salem L, Alberti H, Ben Rayana C, El Atti J, Blouza S, Gaigi S, Achour A, Ben Slama C, Zouari B: Prevalence of metabolic abnormalities in the Tunisian adults: a population based study. Diabetes Metab 2006, 32(2):215-22I.

29. Bustos P, da Silva AA, Amigo H, Bettiol H, Barbieri MA: Metabolic syndrome in young adults from two socioeconomic Latin American settings. Nutr Metab Cardiovasc Dis 2006, I 7(8):58I-9.

30. Ramirez-Vargas E, Arnaud-Vinas MdR, Delisle H: Prevalence of the metabolic syndrome and associated lifestyles in adult males from Oaxaca, Mexico. Salud Publica de Mexico 2007, 49(2):94.

31. Weng XP, Liu YX, Ma J, Wang W, Yang G, Caballero B: An urbanrural comparison of the prevalence of the metabolic syndrome in Eastern China. Public Health Nutr 2007, I O(2): I3 I.

32. Hamman RF, Wing RR, Edelstein SL, Lachin JM, Bray GA, Delahanty L, Hoskin M, Kriska AM, Mayer-Davis EJ, Pi-Sunyer X, et al.: Effect of Weight Loss With Lifestyle Intervention on Risk of Diabetes. Diabetes Care 2006, 29(9):2102-2107.

33. Laatikainen T, Dunbar J, Chapman A, Kilkkinen A, Vartiainen E, Heistaro S, Philpot B, Absetz P, Bunker S, O'Neil A, et al.: Prevention of Type 2 Diabetes by lifestyle intervention in an Australian primary health care setting: Greater Green Triangle (GGT) Diabetes Prevention Project. BMC Public Health 2007, 7(1):249-240.

34. Tuomilehto J, Lindstrom J, Eriksson JG, Valle TT, Hamalainen $H$, Ilanne-Parikka P, Keinanen-Kiukaanniemi S, Laakso M, Louheranta A, Rastas M, et al:: Prevention of type 2 diabetes mellitus by changes in lifestyle among subjects with impaired glucose tolerance. N Engl J Med 200I, 344( ( 8): I343-1350.

35. Australian Bureau of Statistics: Census of Population and Housing: Basic Community Profiles. Canberra: ABS 200I.

36. Healy GN, Dunstan DW, Salmon JO, Shaw JE, Zimmet PZ, Owen N: Television Time and Continuous Metabolic Risk in Physically Active Adults. Medicine \& Science in Sports \& Exercise 2008, 40(4):639.

37. Strath S, Swartz A, Parker S, Miller N, Cieslik L: Walking and metabolic syndrome in older adults. JPAH 2007, 4(4):397-4I0.

38. Xin L, Miller YD, Burton NW, Brown WJ: A preliminary study of the effects of Tai Chi and Qigong medical exercise on indicators of metabolic syndrome and glycaemic control in adults with elevated blood glucose. Br J Sports Med 2008 in press.

39. Blair SN, Brodney S: Effects of physical inactivity and obesity on morbidity and mortality: current evidence and research issues. Med Sci Sport \& Ex 1999, 3 I (I I Suppl):S646-662.

\section{Pre-publication history}

The pre-publication history for this paper can be accessed here:

http://www.biomedcentral.com/1471-2458/9/273/pre pub

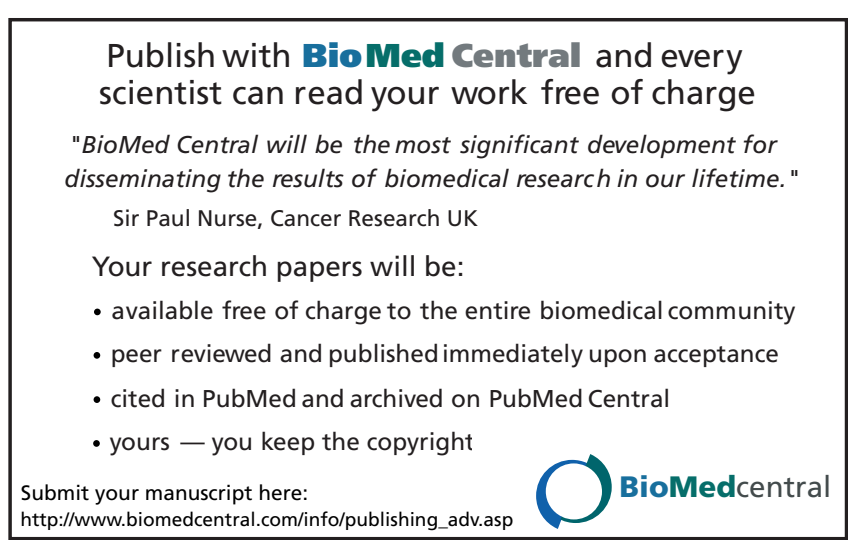

